\title{
Use of quaternised methacrylate polymers and copolymers as catalysts and structure directors for the formation of silica from silicic acid
}

\author{
Yimei Jia, ${ }^{a}$ Graham M. Gray, ${ }^{a}$ John N. Hay, ${ }^{* a}$ Yuting Li, ${ }^{b}$ Gian-Franco Unali, ${ }^{b}$ Fiona L. Baines ${ }^{b}$ and \\ Steven P. Armes $\dagger^{b}$
}

Received 9th December 2004, Accepted 17th March 2005

First published as an Advance Article on the web 7th April 2005

DOI: $10.1039 / b 418372 \mathrm{j}$

\begin{abstract}
A range of quaternised tertiary amine methacrylate-based homopolymers and copolymers were synthesised as mimics of the biopolymers implicated in biosilica formation. These synthetic polymers were evaluated for their ability to catalyse and direct the structure of silica formed by condensation of silicic acid in aqueous solution and at neutral $\mathrm{pH}$. Homo- and co-polymers of differing degrees of quaternisation were studied, while some of the homopolymers also differed in their chain length. All polymers acted as catalysts for the condensation reaction, but at different rates according to their architecture and degree of quaternisation. The resulting silica-polymer hybrids were characterised fully, as were pure silicas obtained by calcination of the hybrids. Some crystallites were present in the hybrids and differences in crystal structure were observed in the calcined silicas, depending on the structure of the polymer, indicating that the polymers exert a structure-directing effect during initial silica formation. The work provides some new insights into structural factors affecting silica growth catalysed by synthetic cationic polymers.
\end{abstract}

\section{Introduction}

A variety of biological organisms (such as diatoms, sponges and grasses) are capable of incorporating silica in vivo at ambient temperature and neutral $\mathrm{pH}$. In the case of diatoms this biosilicification of silica utilises monosilicic acid, $\mathrm{Si}(\mathrm{OH})_{4}$, as the starting material to create silicas with outstanding control over morphology. ${ }^{1-3}$ Recently researchers have isolated the proteins responsible for silica precipitation and a number of proteins, polypeptides and synthetic polymers have been found to precipitate ordered and disordered silica structures from either tetraalkoxysilanes or sodium silicate. ${ }^{3-16}$ It has been postulated that the more pronounced effect of peptides compared to amines is not just due to the many electrostatic interactions or hydrogen bonds available within the polymer but that the relative arrangement of the amino groups is also important. Within a single molecule the electrostatic and/or hydrogen bond interactions are not thought to be sufficient to favour condensation. ${ }^{10,12}$

We have previously reported the use of polyviologens as potential catalysts for monosilicic acid polymerisation. ${ }^{16}$ Little difference was observed with changes in counter-ion and alkyl spacer groups used. Although polyviologens are effective catalysts for monosilicic acid polymerisation it is not possible to apply the variety of counter-ion and alkyl chain spacers we have previously employed during the synthesis of sol-gel hybrids as the solubility of the polymers in the aqueous buffer is a limitation. ${ }^{16}$ However, the work stimulated our interest in the catalytic and structure-directing effects of other structurally well-defined cationic polymers. The use of readily

$\uparrow$ Current address: Department of Chemistry, University of Sheffield, Sheffield, S3 7HF, UK.

*j.hay@surrey.ac.uk accessible synthetic polymers to produce structurallycontrolled silicas offers an attractive alternative to the use of natural or synthetic proteins. The use of well defined block copolymers with an anionic block has been shown to control the crystal formation of barium sulfate. ${ }^{17}$ In Nature, the mediation of biomineralisation processes by amphiphilic macromolecules is increasingly well understood. ${ }^{18}$ Here, we investigate a series of quaternised 2-(dimethylamino)ethyl methacrylate (DMAEMA) homopolymers as well as welldefined block copolymers of DMAEMA with methyl methacrylate (MMA) with varying degrees of quaternisation at a fixed chain length, and fully quaternised but with varying chain length (Fig. 1). The effectiveness of these polymers as catalysts for monosilicic acid polymerisation as well as the nature of the hybrids and final silicas formed is described here.

\section{Experimental}

\section{Materials and techniques}

Sodium silicate solution $(27 \% \mathrm{Si})$ was purchased from Reidel de Haën and used as received. All other chemicals purchased were analytical grade or better and used as received. The $\mathrm{pH} 7.2$ buffer used was Tris- $\mathrm{HCl}$, prepared from tris(hydroxymethyl)aminomethane (Acros). ${ }^{12}$
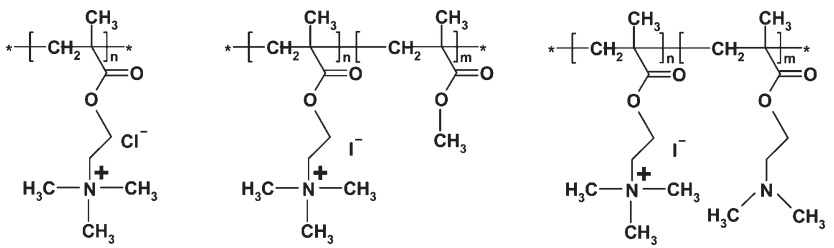

Fig. 1 Quaternised PDMAEMA (left), its block copolymer with MMA (centre) and the partially quaternised PDMAEMA statistical copolymer (right). 
Table 1 Quaternised PDMAEMA homopolymers (the first three entries were obtained by quaternisation of GTP-synthesised PDMAEMA homopolymer with methyl iodide, while the last three entries were synthesised by the atom transfer radical polymerisation of methyl chloride-quaternised DMAEMA monomer)

\begin{tabular}{lcc}
\hline Abbreviation & $\bar{M}_{\mathrm{n}}$ of precursor & $\%$ Quaternisation \\
\hline PDMAEMA-35 & 6800 & 35 \\
PDMAEMA-63 & 6800 & 63 \\
PDMAEMA-100 & 6800 & 100 \\
QDMA $_{30}$ & 4800 & 100 \\
QDMA $_{60}$ & 9600 & 100 \\
QDMA $_{100}$ & 15900 & 100 \\
\hline
\end{tabular}

Infrared spectra were recorded as $\mathrm{KBr}$ pellets on a Perkin Elmer system 2000 FT-IR spectrometer. Solid-state ${ }^{13} \mathrm{C}$ crosspolarized (CP) and ${ }^{29} \mathrm{Si}$ directly polarized (DP) nuclear magnetic resonance (NMR) spectroscopy was carried out at the University of Durham. The spectra were recorded on a Varian Unity Inova spectrometer equipped with a $7.5 \mathrm{~mm}$ magic angle spinning (MAS) probe. The ${ }^{29} \mathrm{Si}$ DP quantitative spectra were measured at ambient temperature using a $90^{\circ}$ pulse angle, a $120.0 \mathrm{~s}$ recycle, an acquisition time of $15.0 \mathrm{~ms}$, a spin-rate of $c a .5000 \mathrm{~Hz}$ and a frequency of $59.58 \mathrm{MHz}$ (ca. 450 repetitions). The ${ }^{13} \mathrm{C} \mathrm{CP}$ spectra were obtained at ambient temperature using an acquisition time of $30.0 \mathrm{~ms}$, a pulse delay of $2.0 \mathrm{~s}$, a spin-rate of $c a .4800 \mathrm{~Hz}$ and a frequency of $75.43 \mathrm{MHz}$ ( $c a .1000$ repetitions). The conventional $\mathrm{Q}^{n}$ notation is used to denote tetrafunctional silica species, where $n$ is the number of siloxane groups bonded to the silicon atom.

Powder X-ray diffraction (XRD) analysis was performed on a Seifert XRD 3003 TT powder diffractometer using $\mathrm{Cu} \mathrm{K} \alpha$ radiation. Scanning electron micrographs were recorded on a Hitachi S-4000 or a Hitachi S-3200N scanning electron microscope. The Hitachi S-3200N was also used to acquire energy dispersive X-ray spectrometry (EDS) data. Transmission electron micrographs and electron diffraction data were recorded on a Philips EM 400T transmission electron microscope.

\section{Polymer synthesis}

The samples of PDMAEMA homopolymer and PDMAEMAPMMA diblock copolymer were prepared by group transfer polymerisation (GTP) in anhydrous THF at ambient temperature, as described previously. ${ }^{19}$ Partially quaternized PDMAEMA homopolymers and PDMAEMA-PMMA diblock copolymers were prepared by reaction with substoichiometric quantities of methyl iodide, as described by Vamvakaki et $a l .^{20}$ The fully quaternised PDMAEMA homopolymers were prepared by atom transfer radical polymerisation (ATRP) of methyl chloride-quaternised DMAEMA monomer as described previously. ${ }^{21}$ Note that the halide counter-ions differ for the homopolymers prepared by the different routes (chloride $v s$. iodide).

Characterisation data for all the quaternised polymers are presented in Tables 1 and 2. The abbreviations used to describe these samples throughout this paper are presented in column one of Tables 1 and 2.

\section{Silica precipitation}

Monosilicic acid polymerisation was carried out using different protocols. Some reactions were performed with varying polymer concentrations to maintain an equivalent amount of quaternary ammonium groups across the polymer series, while in others the polymer concentration was kept constant and the degree of quaternisation varied. It was envisaged that this would help to reveal differences in the rate of monosilicic acid polymerisation resulting from architectural effects of the homo- or block co-polymers such as the presence or absence of a hydrophobic unit and the length of the hydrophilic or hydrophobic block which might be expected to affect the conformation adopted in solution. In addition, some reactions were performed with a specific polymer at different concentrations to see if there was any effect of concentration upon the reaction beyond the expected increase in rate as the proportion of quaternary nitrogen groups increases.

The method used was that employed by Coradin and Livage. ${ }^{12}$ Molybdosilicic acid titration was used to follow the rate of reaction. ${ }^{22}$ Sodium silicate solution $(0.10 \mathrm{ml})$ was added to the buffer solution $(30 \mathrm{ml})$ in a $50 \mathrm{ml}$ conical flask. The mixture was stirred for $10 \mathrm{~min}$ before a solution of polymer in buffer $(3 \mathrm{ml})$ was added. This represents a $c a .0 .0047 \mathrm{M}$ monosilicic acid solution. Stirring was maintained throughout the reaction. Periodically aliquots $(400 \mu \mathrm{l})$ of the reaction mixture were taken and added to deionised water $(5 \mathrm{ml})$. Sulfuric acid $(200 \mu \mathrm{l}, 1.5 \mathrm{M})$ and ammonium molybdate $(400 \mu \mathrm{l}, 0.08 \mathrm{M})$ were added and the mixture left to stand for $10 \mathrm{~min}$ before the UV absorption of the solution at $400 \mathrm{~nm}$ was recorded. After $2 \mathrm{~h}$ the reaction mixture was centrifuged, and the recovered solids dried under a flow of air at room temperature overnight.

The polymer-silica hybrids were calcined in air at $650{ }^{\circ} \mathrm{C}$ for $24 \mathrm{~h}$ in a muffle furnace.

\section{Results and discussion}

The polymers were synthesised as described previously using GTP and ATRP; ${ }^{19-21}$ these two techniques ensure reasonably good control over the molecular weight and molecular weight distribution. The choice of polymers was based on the known effect of cationic proteins and other cationic polymers in natural biosilicification. ${ }^{1-16}$ It is also known more generally in biomineralisation that auxiliary macromolecules play a rôle

Table 2 MMA-DMAEMA block copolymers quaternised with MeI

\begin{tabular}{lllr}
\hline Abbreviation of copolymer & Composition MMA : DMAEMA (mol \%) & $\bar{M}_{\mathrm{n}}$ of precursor & \% Quaternisation \\
\hline MMA-DMAEMA-16 & $21: 79$ & 9400 & 16 \\
MMA-DMAEMA-23 & $21: 79$ & 9400 & 23 \\
MMA-DMAEMA-45 & $21: 79$ & 9400 & 45 \\
MMA-DMAEMA-100 & $21: 79$ & 9400 & 100 \\
\hline
\end{tabular}


in controlling the structure of the growing mineral ${ }^{1,2,23}$ and it was thus of interest to compare the behaviour of the quaternised PDMAEMA homopolymers with that of the DMAEMA-MMA block copolymers, where the PMMA block can potentially exert a non-catalytic auxiliary effect. It has further been shown that the hydrophobic PMMA block leads to self-assembly of the block copolymers in aqueous solution, ${ }^{24}$ which allows the possible effect of using copolymer micelles with cationic coronas (rather than molecularly dissolved cationic polymers) on silica catalysis to be explored. It is anticipated that the precise structure of the polymer in solution will itself be influenced by the presence of the continually changing silica species (in a similar way that silicon precursor induced surfactant mesophase formation has been known for some time $\mathrm{e}^{25,26}$ ), but a detailed study of this behaviour is beyond the scope of the current investigation.

\section{Monosilicic acid polymerisation}

Whilst a variety of precursors can act as the silica source, the choice of silicic acid provides a comparison with an earlier study, ${ }^{12}$ while being directly relevant to the situation found in Nature. ${ }^{4}$ The Tris- $\mathrm{HCl}$ buffer is chosen to avoid the presence of phosphate, which can interact with the silica. A kinetic study of silica formation using the molybdosilicic acid titration method provides a facile way of determining the catalytic effects of the various synthetic polymers examined in this study. The method used was analogous to that described by Coradin and Livage ${ }^{12}$ in their peptide study, although the concentration of silicic acid was increased for practical reasons, but still within the accepted limits of the method. ${ }^{22}$ Despite the various differences in the results of monosilicic acid polymerisation (discussed below) the results for all the polymers used share several features in common. In the absence of polymer, the addition of sodium silicate solution to the buffer did not result in any visible precipitation and the solution remained clear for at least $8 \mathrm{~h}$. Nonetheless, a gradual decrease in silicic acid concentration was observed with time (Fig. 2), suggesting the formation of soluble oligomeric species. This is entirely as expected for silicic acid

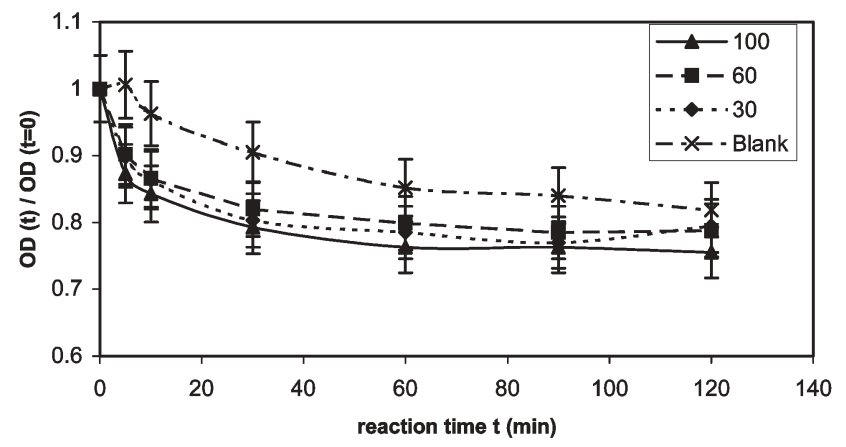

Fig. 2 Evolution with time of monosilicic acid content as measured by the molybdosilicic acid method in the presence of fully quaternised PDMAEMA (QDMA) with varying nominal chain lengths at a constant concentration of quaternary ammonium groups at pH 7.2. Polymer : silica molar ratios $1.07 \times 10^{-3}$ (QDMA-100; $\left.\mathbf{\Delta}\right), 1.79 \times$ $10^{-3}$ (QDMA-60; ) $3.58 \times 10^{-3}$ (QDMA-30; $)$. Blank $(\times)=$ polymer-free system. condensation at neutral $\mathrm{pH}$. The observed decrease in $\mathrm{Si}(\mathrm{OH})_{4}$ concentration is slightly greater than that found previously, ${ }^{12}$ perhaps reflecting the increased starting concentration, as a still greater effect has been observed with an even higher silicic acid initial concentration and poly(allylamine hydrochloride) as catalyst. ${ }^{10}$

The addition of polymer to the silicic acid mixture immediately results in the solution becoming turbid and the formation of a visible precipitate is evident shortly thereafter (several minutes). A sharp decrease in the monosilicic acid concentration ( $c a .10 \%)$ is observed for all polymers within the first few minutes, in contrast to the blank (polymer-free) sample which shows a slow and steady decrease in concentration (Fig. 2). Subsequently the curve flattens out and resembles that obtained in the absence of polymer. The initial decrease can be attributed to the precipitation of polysilicic acid caused by the electrostatic and hydrogen bonding interactions with the cationic polymer. After precipitation the polymer becomes increasingly entrapped within the growing silica precipitate until it is unable to exert any catalytic effect on the remaining monosilicic acid in solution. As the catalytic effect weakens, any further silica formation will proceed at a much lower rate typical of the polymer-free reaction. Hence the rate of decrease in silicic acid concentration slows and eventually resembles that observed in the absence of polymer, although differences are observed in the final silicic acid concentration. The silica products are true polymer-silica hybrids, in the same way that natural silica exoskeletons are proteinaceous hybrids. The ability of the polymers to induce monosilicic acid polymerisation remains effective for up to $30 \mathrm{~min}$. Foaming was observed for the amphiphilic block copolymer PMMA-PDMAEMA100. Because of the surfactancy of these polymers, this was not unexpected but it is not clear how much this affected the results obtained.

PDMAEMA homopolymer at varying degrees of quaternisation and chain lengths. At a constant concentration of quaternary ammonium groups no differences beyond experimental error were observed for the fully quaternised polymer (QDMA) at varying chain lengths, as would be expected if the primary controlling factor is the concentration of catalytic species (Fig. 2). At best, the final degree of condensation is slightly higher than for the uncatalysed case and comparable to the degree of condensation observed for polylysine at the same $\mathrm{pH} .{ }^{12}$ At this $\mathrm{pH}$, the polypeptide also carries a positive charge, resulting in electrostatic interactions with growing silicate species, as expected for QDMA.

At a fixed polymer concentration, however, after the initial sharp drop in $\mathrm{Si}(\mathrm{OH})_{4}$ concentration in all cases, the longer chain length $\left(\mathrm{QDMA}_{100}\right)$ polymer appears to polymerise monosilicic acid more slowly than observed for the shorter chain lengths $\left(\mathrm{QDMA}_{30,60}\right)$ as shown in Fig. 3. This results in an overall lower degree of condensation in the case of QDMA $_{100}$. This is somewhat surprising when it is considered that there are more quaternised ammonium groups present overall in the longer chain length polymer. This could be related to the slower micro-diffusion rate of the longer coiled polymer chains in the local environment of the polymerising silicate species. It may also reflect a greater propensity for this 


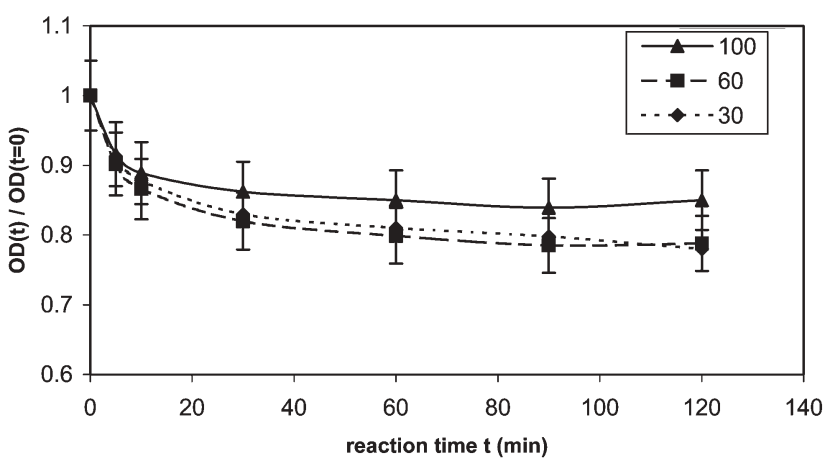

Fig. 3 Evolution with time of monosilicic acid content as measured by the molybdosilicic acid method in the presence of fully quaternised PDMAEMA (QDMA) with varying nominal block lengths (100, $\mathbf{\Delta}$; $60, \mathbf{\square} ; 30, \bullet)$ at a constant polymer concentration $\left(3.38 \times 10^{-5} \mathrm{M}\right)$ at pH 7.2. Polymer : silica molar ratio $1.79 \times 10^{-3}$.

chain to bind electrostatically to the silicate species, leading to more rapid shielding of the catalytic positive charge.

Furthermore a decrease in concentration of QDMA $_{100}$ results in an increase in the rate of monosilicic acid polymerisation and a reduction in the overall final amount of monosilicic acid as shown in Fig. 4. The amount of unreacted silicic acid remaining at the higher polymer concentration is actually greater than for the polymer-free sample, although the overall extent of reaction is greater for the lower concentration of polymer. This is in marked contrast to the results obtained for $\mathrm{QDMA}_{30}$ which, although on the edge of experimental error, appear to show an increase in the rate of monosilicic acid polymerisation with an increase in concentration and a small increase in extent of reaction compared to the polymerfree case (Fig. 4). This would be expected from the increase in concentration of catalytic groups. The results observed for PDMAEMA homopolymers at varying degrees of quaternisation (Fig. 5) appear to support the idea of the polymer architecture having an effect. Surprisingly, PDMAEMA100 and PDMAEMA-35 polymerise monosilicic acid at a markedly faster rate than that observed with the PDMAEMA63 polymer. In addition, while the overall extent of silicic acid

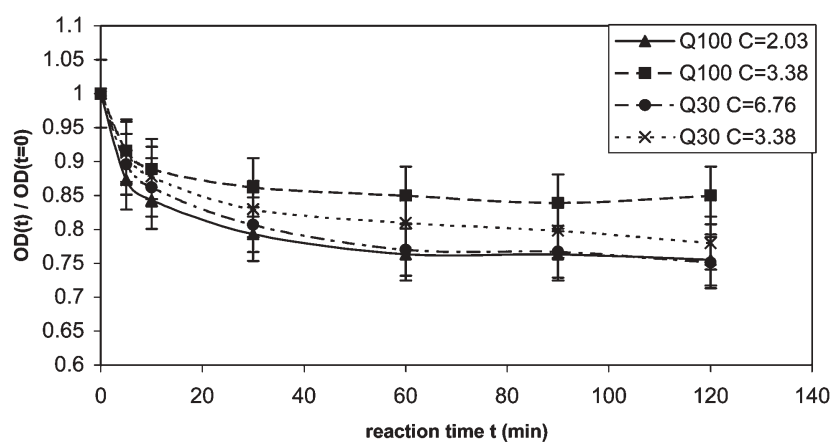

Fig. 4 Evolution with time of monosilicic acid content as measured by the molybdosilicic acid method in the presence of QDMA $_{100}$ at two different polymer concentrations $\left(2.03, \boldsymbol{\Lambda}\right.$, and $\left.3.38 \times 10^{-5} \mathrm{M}, \boldsymbol{\square}\right)$ and QDMA $_{30}$ at two different polymer concentrations $(3.38, \times$, and $\left.6.76 \times 10^{-5} \mathrm{M}, 0\right)$ at $\mathrm{pH} 7.2$. Polymer : silica molar ratios respectively $1.07 \times 10^{-3}, 1.79 \times 10^{-3}, 1.79 \times 10^{-3}, 3.58 \times 10^{-3}$.

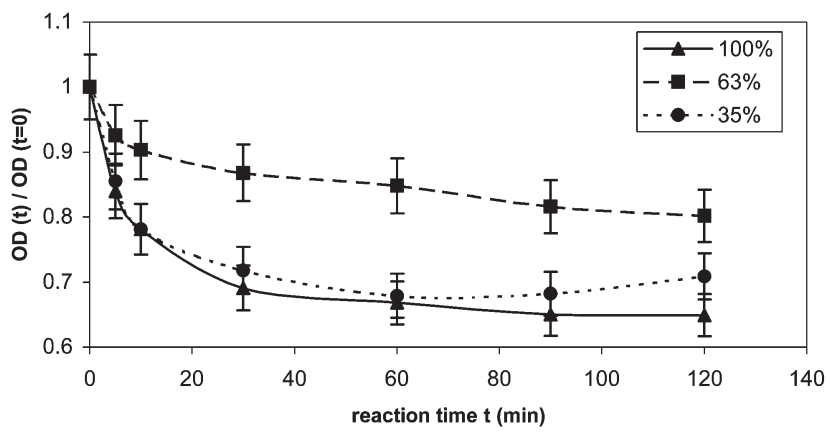

Fig. 5 Evolution with time of monosilicic acid content as measured by the molybdosilicic acid method in the presence of PDMAEMA at varying degrees of quaternisation $(100 \%, \mathbf{\Delta} ; 63 \%, \mathbf{\square} ; 35 \%, \bullet)$ at $\mathrm{pH}$ 7.2. Polymer concentration $8.91 \times 10^{-5} \mathrm{M}$, polymer : silica molar ratio $4.71 \times 10^{-3}$.

reaction in the case of PDMAEMA-63 is comparable to the polymer-free case, the extent of reaction is much greater for the other polymers, which are also more effective than polylysine at this $\mathrm{pH}$ and comparable to polylysine at $\mathrm{pH} 9.2$, where the proportion of negatively charged silicate species is higher. $^{12}$ These apparently counter-intuitive results could arise from differences in the solution conformation of the polymers, affecting the accessibility and catalytic efficiency of the cationic sites. A detailed in situ study of polymer conformation through the course of the reactions would be instructive, but is beyond the scope of this preliminary study.

MMA-DMAEMA block copolymers at varying degrees of quaternisation. The results for these polymers are largely within experimental error (Fig. 6) although it does appear that the polymers fall within two 'groups'. Copolymers with higher degrees of quaternisation (MMA-DMAEMA-100, MMADMAEMA-45 and MMA-DMAEMA-23) are in good agreement with each other, whereas the polymer with the lowest degree of quaternisation (MMA-DMAEMA-16) appears to polymerise monosilicic acid at a slightly lower rate (although this is on the edge of experimental error). The less marked catalytic effect of the PMMA-PDMAEMA-16 diblock copolymer is also reflected in the overall extent of reaction,

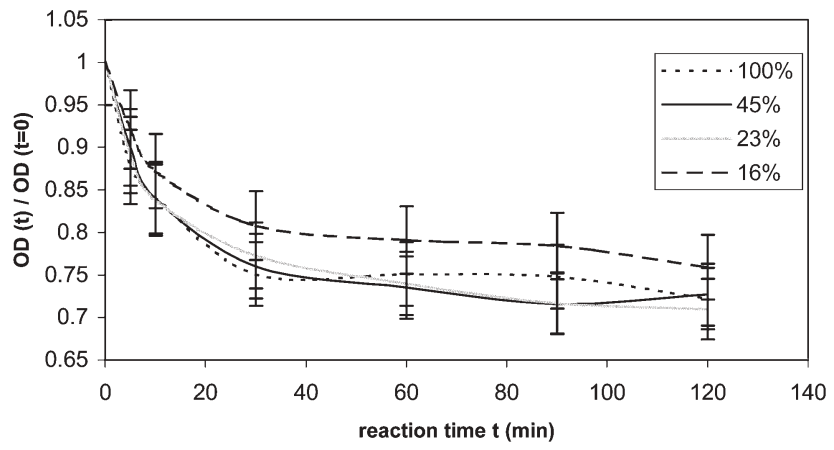

Fig. 6 Evolution with time of monosilicic acid content as measured by the molybdosilicic acid method in the presence of PDMAEMAPMMA at varying degrees of quaternisation $(100,45,23$ and $16 \%$ ) at $\mathrm{pH}$ 7.2. Polymer concentration $6.45 \times 10^{-5} \mathrm{M}$, polymer : silica molar ratio $3.41 \times 10^{-3}$. 
which is lowest for PMMA-PDMAEMA-16, although still greater than the polymer-free case for all copolymers. Such a difference between the $16 \%$ and $23 \%$ quaternised polymers would not be expected on the basis of the difference in concentration of catalytic groups alone. Inaccessibility of the catalytic groups seems unlikely to be the reason for these results, as the PMMA block should lead to micellar selfassembly, with the quaternised DMAEMA groups accessible on the periphery of the micelle corona. One possible rationale is the effect of the $84 \%$ non-quaternised basic tertiary amine groups, which could act as an inhibitor of silica formation through local buffering of solution $\mathrm{pH}$ to a value different to that of the bulk solution $\mathrm{pH}$.

\section{Polymer-silica hybrid products}

All the polymer-silica hybrids were obtained as white solids and found to be amorphous by powder XRD. No evidence was found for any specific ordering induced by the presence of the polymers. FTIR analysis revealed the characteristic $\mathrm{C}=\mathrm{O}$ stretch at $1670-1780 \mathrm{~cm}^{-1}$ in all the hybrids, confirming the presence of polymer. This is consistent with the kinetic studies, which provide indirect evidence for encapsulation of the polymers within the growing silica network. Absorptions due to $\mathrm{Si}-\mathrm{O}-\mathrm{Si}$ bending and $\mathrm{Si}-\mathrm{OH}$ vibration at 800 and $950 \mathrm{~cm}^{-1}$ respectively confirmed the incorporation of silica. A broad absorption at $c a \cdot 1000-1220 \mathrm{~cm}^{-1}$ is most likely composed principally of the $\mathrm{Si}-\mathrm{O}$ stretch with an additional component from the $\mathrm{C}-\mathrm{O}$ stretch.

Nuclear magnetic resonance spectroscopy. Analysis by ${ }^{13} \mathrm{C}$ solid state NMR spectroscopy of the hybrids containing PDMAEMA-35, PDMAEMA-63 and PDMAEMA-100 confirmed the incorporation of polymer into the hybrids, the spectra showing resonances consistent with the polymer structures. NMR spectroscopy also revealed that, although a significant difference was noted in the rate of monosilicic acid polymerisation with PDMAEMA-63 compared to the other homopolymers (which both result in faster condensation), it is only the fully quaternised polymer (PDMAEMA-100) that shows any significant difference in the hybrid structure by ${ }^{29} \mathrm{Si}$ NMR (Fig. 7). An increase in the amount of $Q^{4}$ species relative to $\mathrm{Q}^{3}$ suggests that using PDMAEMA-100 leads to a more fully condensed silica network. This makes sense if the

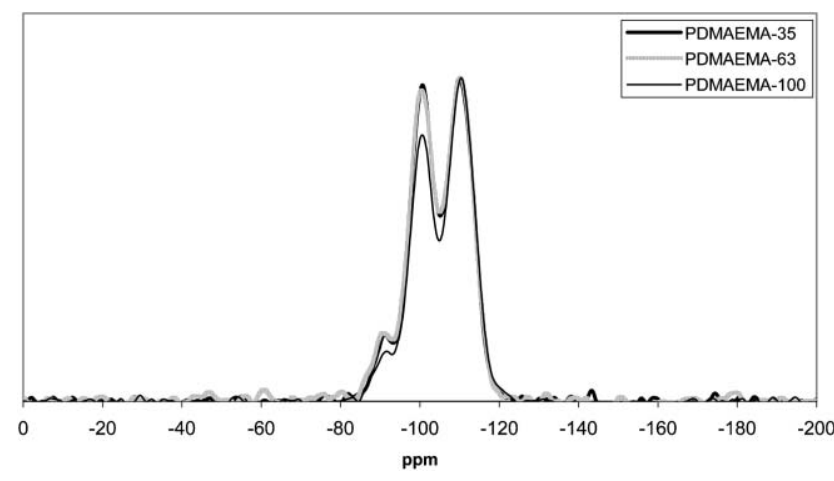

Fig. 7 Direct polarisation ${ }^{29} \mathrm{Si}$ MAS NMR of hybrids containing PDMAEMA-35, PDMAEMA-63 and PDMAEMA-100. catalytic effect is related not only to the accessibility of the trimethylammonium groups but also their local concentration on the polymer backbone, which could lead to cooperative effects of several adjacent catalytic groups in the case of PDMAEMA-100, but not PDMAEMA-35. It is emphasised that the kinetic effects described earlier refer only to the initial reduction in the concentration of the silicic acid, but not to the extent of subsequent condensation. These results demonstrate that the factors which influence the initial condensation of silicic acid are not necessarily identical to those which control the later stages of condensation in these silicates.

Electron microscopy. In general the hybrids consist of aggregates of roughly spherical particles of varying size (which are for the most part below $0.1 \mu \mathrm{m}$ ). No significant differences in particle size were observed for hybrids formed from various polymer architectures or differing degrees of quaternisation. The surface morphology of the aggregates varied from fairly smooth to relatively rough and it was not possible to rationalise the differences, if any, between samples. Energy dispersive X-ray spectrometry (EDS) revealed that the hybrids are composed mainly of silica. In addition to the expected signal from the polymer, trace amounts of sodium and chlorine (primarily from the buffer solution) are also present. Elemental mapping reveals that the various elements are mostly distributed fairly evenly throughout the samples except in rough areas where it appears that sodium and chlorine predominate (Fig. 8). It is not unreasonable that this is due to the formation of salt crystals; however, if they are present it is in sufficiently low quantities for the hybrids to remain amorphous by powder XRD. It is not clear whether the images observed under SEM accurately reflect the material as it is formed in situ rather than the effects of aggregation throughout the reaction and the subsequent drying process in a petri dish. Therefore, immediately after the addition of polymer to the silica solution, aliquots of the cloudy suspension (containing precipitating material) were removed using a pipette, placed onto holey carbon film and allowed to dry under a flow of air. The samples were then investigated using transmission electron microscopy (TEM) in order to better understand the in situ silica formation process.

Analysis of the hybrids by TEM revealed them to consist predominantly of amorphous roughly spherical particles in accordance with the results obtained from SEM; however, other particle shapes were also observed in varying quantities (Fig. 9). These shapes were regular and subsequent analysis revealed that a variety of regularly shaped 'microcrystalline' species were present in many of the hybrids. These were typically found to be tetragonal in shape (Fig. 10, left) but rodlike species (Fig. 9) were also observed. Diffraction patterns were collected and confirmed that these species were crystalline (Fig. 10, right) and that all possessed a face centred cubic structure, although the actual lattice parameters varied somewhat (Tables 3 and 4). Although, as noted above, EDS showed that sodium and chlorine are present in significant amounts in certain areas, none of the lattice parameters analysed corresponded to the $\mathrm{NaCl}$ structure. The formation of multiple morphologies may arise from kinetically-controlled crystal growth mediated by the presence 

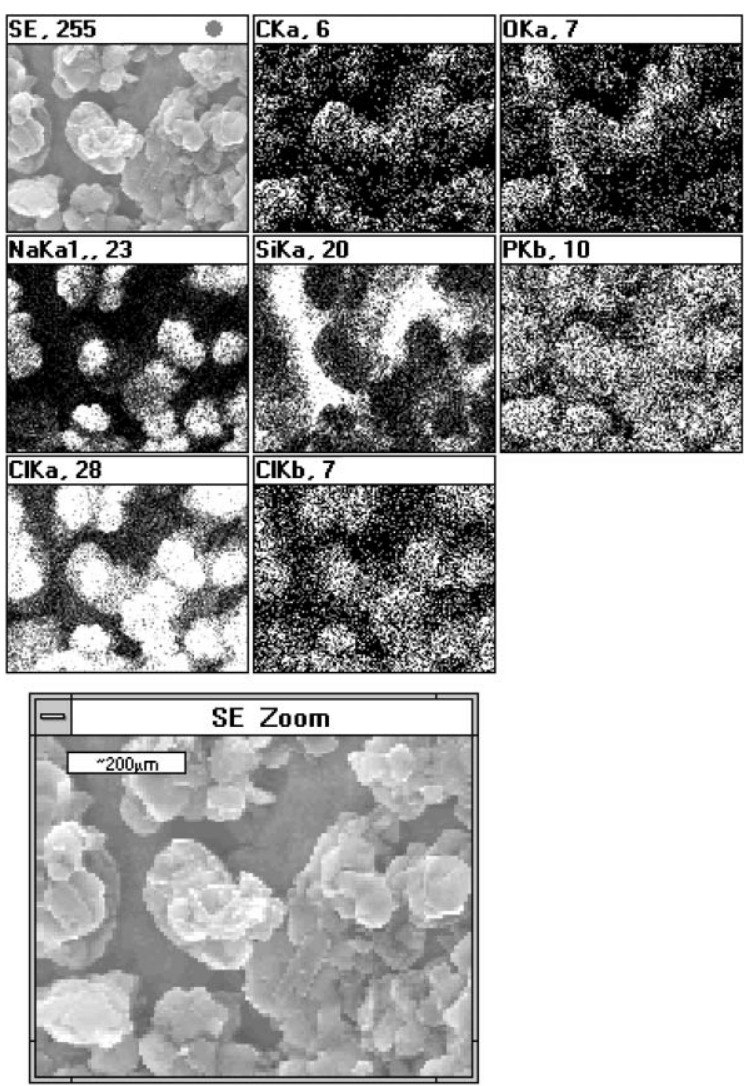

Fig. 8 EDS mapping image shows $\mathrm{Na}^{+}$and $\mathrm{Cl}^{-}$are the main components of the surface particles in the hybrid formed from QDMA $_{30}$ $[\mathrm{SE}=$ normal micrograph $]$

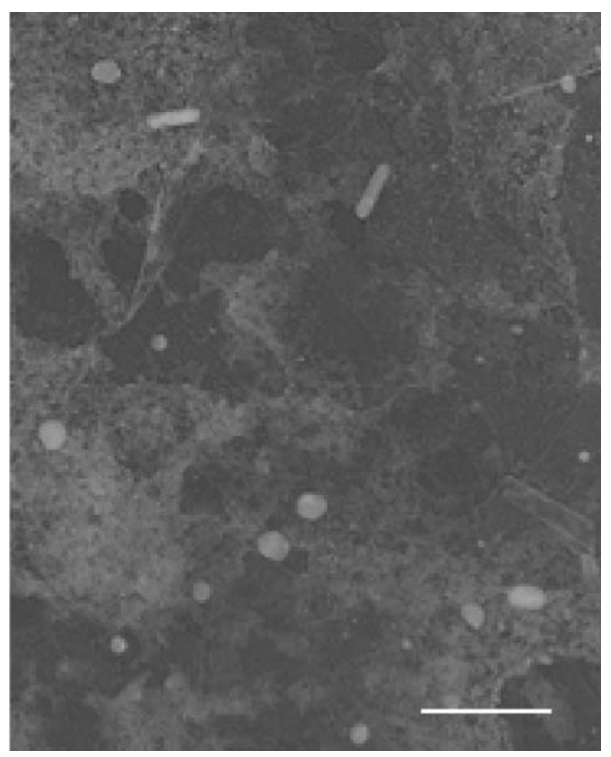

Fig. 9 TEM image shows nanoparticles with different shapes in the hybrid containing MMA-DMAEMA-100 (scale bar $=0.27 \mu \mathrm{m})$.

of the organic polymer matrix and involving polymer conformational changes during the silicification process. ${ }^{18}$ It has been found that biogenic inorganic oxides can display a variety of morphologies not necessarily accessible by conventional chemical synthesis. ${ }^{27}$

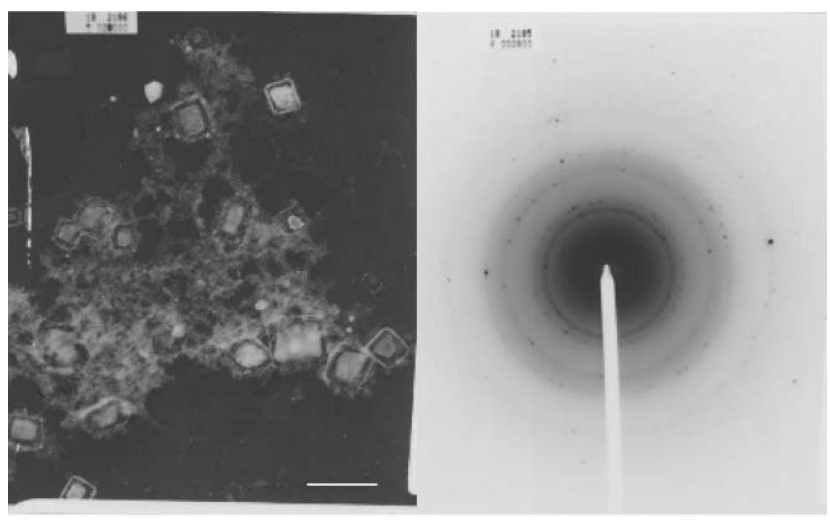

Fig. 10 TEM image (left) shows the occurrence of regular tetragonal shapes in the hybrid containing MMA-DMAEMA-100 (scale bar = $0.45 \mu \mathrm{m})$. The X-ray diffraction pattern of these particles (right) confirms that they are crystalline.

Table 3 Diffraction pattern analysis of microcrystallites found in hybrids incorporating $\mathrm{QDMA}_{100}$

\begin{tabular}{llllll}
\hline$R / \mathrm{mm}$ & $R^{2}$ & $R^{2} / R_{1}{ }^{2}$ & $3\left(R^{2} / R_{1}{ }^{2}\right)$ & $\begin{array}{l}\text { Possible value } \\
\text { for } h^{2}+k^{2}+l^{2}\end{array}$ & Index \\
\hline 7.8 & 60.84 & 1 & 3 & 3 & $(111)$ \\
9.0 & 81 & 1.332 & 3.994 & 4 & $(200)$ \\
12.8 & 163.8 & 2.693 & 8.079 & 8 & $(220)$ \\
15 & 225 & 3.698 & 11.095 & 11 & $(222)$ \\
15.6 & 243.4 & 4 & 12 & 12 & $(311)$ \\
\hline
\end{tabular}

Table 4 Diffraction pattern analysis of microcrystallites found in hybrids incorporating MMA-DMAEMA-45

\begin{tabular}{llllll}
\hline$R / \mathrm{mm}$ & $R^{2}$ & $R^{2} / R_{1}{ }^{2}$ & $3\left(R^{2} / R_{1}{ }^{2}\right)$ & $\begin{array}{l}\text { Possible value } \\
\text { for } h^{2}+k^{2}+l^{2}\end{array}$ & Index \\
\hline 5.9 & 34.81 & 1 & 3 & 3 & $(111)$ \\
6.9 & 47.61 & 1.37 & 4.103 & 4 & $(200)$ \\
9.8 & 96.04 & 2.759 & 8.277 & 8 & $(220)$ \\
11.5 & 132.2 & 3.799 & 11.398 & 11 & $(222)$ \\
11.9 & 141.6 & 4.068 & 12.204 & 12 & $(311)$ \\
\hline
\end{tabular}

\section{Silicas formed by calcination}

Calcination of the hybrids in air at $650{ }^{\circ} \mathrm{C}$ for $24 \mathrm{~h}$ resulted in a mixture of amorphous and crystalline silicas which show some variation depending on polymer architecture and degree of quaternisation. The calcination temperature is below the temperature at which significant phase changes normally occur in silica. ${ }^{22}$ The PDMAEMA homopolymer at varying degrees of quaternisation produced crystalline silicas as shown in Fig. 11. Only the MMA-DMAEMA copolymers at a low degree of quaternisation produced crystalline silicas when calcined, but this crystallinity was less marked than those obtained for the homopolymers.

The powder XRD patterns resulting from the calcination of the hybrid containing PDMAEMA-35 (Fig. 11a), and to a lesser extent that obtained from the MMA-DMAEMA-23, resembles that produced when sodium is not fully removed from sodium silicate solution that is subsequently polymerised. ${ }^{22}$ This is not too surprising, as EDS has shown that sodium is present in the hybrids. What is unusual is that this crystal structure is not observed in all the final silicas. 


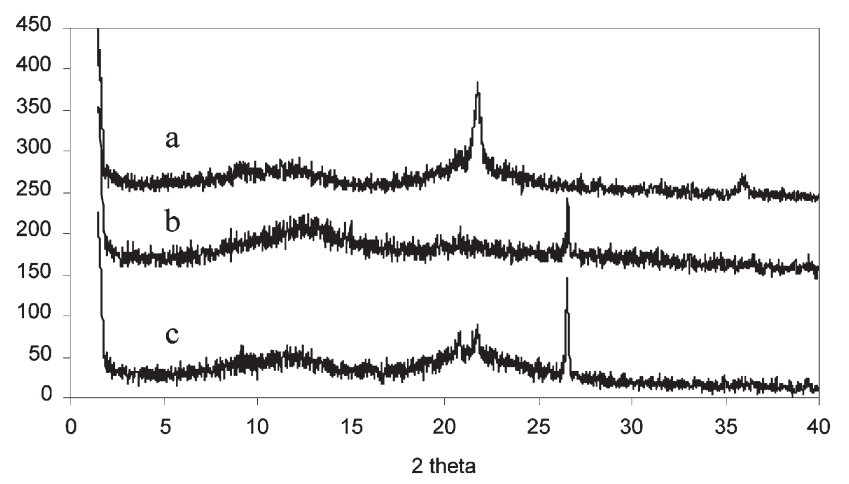

Fig. 11 XRD patterns of (a) PDMAEMA-35, (b) PDMAEMA-63 and (c) PDMAEMA-100 containing hybrids after calcination (arbitrary $y$-axis).

PDMAEMA-63 and PDMAEMA-100 (Figs. 11b and 11c respectively) clearly result in a different crystal structure, as do the other MMA-DMAEMA block copolymers, which form amorphous silica. The apparently anomalous behaviour of the MMA-DMAEMA-100 copolymer is interesting, as its hybrid shows evidence for microcrystalline structures, but none are evident in the calcined silica. Clearly there is an effect of polymer structure on the structure of the final silicas, producing different crystalline or amorphous structures. One unanswered question is whether the anion present in the polymer has an effect on the structure development and subsequent crystallisation. Previous work with cationic ionene polymers saw no evidence for anion effects in the condensation reaction, but the effect on calcinations was not studied. ${ }^{16}$ In the present case, the PDMAEMA- $x$ polymers and the block copolymers contain iodide ions, but the buffer solution contains chloride hence masking any specific anion effect. It would be of interest to investigate such effects in future work by controlling the nature of the anion in both the polymer and the buffer.

For the PDMAEMA homopolymer an increase in the degree of quaternisation may be responsible for an increase in structure control (as discussed above, PDMAEMA-100 has a clear effect upon the silica structure of the hybrid) but the opposite appears to be true for the MMA-DMAEMA copolymer. A combination of electrostatic and topological features is likely to be responsible for the structure-directing effect. The formation of crystalline silicas at such relatively low calcination temperatures is most likely a consequence of the effect of the polymers in producing partial organisation of the silica network in the hybrids and facilitation of further structural reorganisation in the early stages of calcination, prior to thermolysis of the polymer. The extent of structural organisation in the first-formed hybrids is likely to vary according to the spatial separation of the polymer and the silica, with possible formation of some non-templated silica at the surface of individual particles.

\section{Conclusions}

PDMAEMA at various degrees of quaternisation and its block copolymer with methyl methacrylate are effective catalysts for monosilicic acid polymerisation at neutral $\mathrm{pH}$. Differences in polymer architecture have been shown to lead to differences in the rate and extent of polymerisation as well as differences in the structure of the hybrids and silicas formed after calcination. The work points to various competing factors which influence the catalytic activity and structure-directing effects of such synthetic cationic polymers, including polymer solution conformation, micro-environment effects and ease of electrostatic binding between cationic polymer groups and anionic silicate species. A much more detailed study of these effects might lead to sufficient fundamental understanding to ultimately control polymer structure to permit precise tailoring of the final silica network structure. Although the hybrids are mostly amorphous, some microcrystallites are also present in the hybrids immediately after formation. The exact nature of these microcrystallites and their possible involvement in the structure of the final silicas is unclear and warrants further investigation. This will include real time elevated temperature XRD studies.

\section{Acknowledgements}

Financial support from the UK Engineering \& Physical Sciences Research Council (to GG and YTL) is gratefully acknowledged. The authors wish to thank Dr Yanling Chen (MSSU, Surrey) for his assistance in obtaining the TEM images and calculation of the lattice parameters. The authors also thank Dr David Apperley (University of Durham) for provision of solid-state ${ }^{13} \mathrm{C}$ and ${ }^{29} \mathrm{Si}$ NMR services

Yimei Jia, ${ }^{a}$ Graham M. Gray, ${ }^{a}$ John N. Hay, ${ }^{* a}$ Yuting Li, ${ }^{b}$

Gian-Franco Unali, ${ }^{b}$ Fiona L. Baines ${ }^{b}$ and Steven P. Armes $\dagger^{b}$

${ }^{a}$ Chemistry Division, School of Biomedical and Molecular Sciences,

University of Surrey, Guildford, Surrey, UK GU2 $7 X H$.

E-mail: j.hay@surrey.ac.uk

${ }^{b}$ School of Chemistry, Physics and Environmental Science, University of Sussex, Brighton, Sussex, UK

\section{References}

1 D. E. Morse, Trends Biotechnol., 1999, 17, 230.

2 E. G. Vrieling, T. P. M. Beelen, R. A. van Santen and W. W. C. Gieskes, J. Biotechnol., 1999, 70, 39.

3 M. Sumper and N. Kröger, J. Mater. Chem., 2004, 14, 2059.

4 N. Kroger, R. Deutzmann and M. Sumper, Science, 1999, 286, 1129.

5 K. Shimuzi, J. N. Cha, G. D. Stucky and D. E. Morse, Proc. Natl. Acad. Sci. USA, 1998, 95, 6234.

6 Y. Zhou, K. Shimuzi, J. N. Cha, G. D. Stucky and D. E. Morse, Angew. Chem., Int. Ed., 1999, 38, 780.

7 C. C. Perry and T. Keeling-Tucker, Chem. Commun., 1998, 2587.

8 D. Belton, G. Paine, S. V. Patwardhan and C. C. Perry, J. Mater. Chem., 2004, 14, 2231.

9 T. Mizutani, H. Nagase and H. Ogoshi, Chem. Lett., 1998, 133.

10 T. Mizutani, H. Nagase, N. Fujiwara and H. Ogoshi, Bull. Chem. Soc. Jpn, 1998, 71, 2017.

11 J. N. Cha, G. D. Stucky and D. E. Morse, Nature, 2000, 403, 289.

12 T. Coradin and J. Livage, Colloids Surf., B, 2001, 21, 329.

13 T. Coradin, C. Roux and J. Livage, J. Mater. Chem., 2002, 12, 1242.

14 S. V. Patwardhan and S. J. Clarson, Silicon Chem., 2002, 1, 207.

15 T. Coradin and J. Livage, Langmuir, 2002, 18, 2331.

16 G. M. Gray and J. N. Hay, Mater. Res. Soc. Symp. Proc., 2003, $775,179$.

17 K. L. Robinson, J. V. M. Weaver, S. P. Armes, E. D. Marti and F. C. Meldrum, J. Mater. Chem., 2002, 12, 890.

18 S. Mann, Biomineralization. Principles and Concepts in Bioinorganic Materials Chemistry, Oxford University Press, Oxford, 2001. 
19 F. L. Baines, N. C. Billingham and S. P. Armes, Macromolecules, 1996, 29, 3416.

20 M. Vamvakaki, G. F. Unali, V. Bütün, S. Boucher, K. L. Robinson, N. C. Billingham and S. P. Armes, Macromolecules, 2001, 34, 6839.

21 Y. Li, X. Jin, S. Zhu and S. P. Armes, Macromolecules, 2003, 36, 8268.

22 R. K. Iler, The Chemistry of Silica: Solubility, Polymerization, Colloid and Surfaces Properties, and Biochemistry, Wiley, New York, 1979.
23 V. Martin-Jézéquel, M. Hildebrand and M. A. Brzezinski, J. Phycol., 2000, 36, 821.

24 F. L. Baines, S. P. Armes, N. C. Billingham and Z. Tuzar, Macromolecules, 1996, 29, 8151.

25 G. S. Attard, J. C. Glyde and C. G. Göltner, Nature, 1995, 378, 366.

26 N. K. Raman, M. T. Anderson and C. J. Brinker, Chem. Mater., $1996,8,1682$

27 E. Bäuerlein, Angew. Chem., Int. Ed., 2003, 42, 614. 